\title{
Impedance Characteristics of Some Experimental Broad-Band Antennas for Vertical Incidence Ionosphere Sounding
}

\author{
By H. N. Cones
}

\begin{abstract}
This paper presents the results of measurements of the modulus of input impedance of a number of nonresonant antennas over a continuous frequency range from 1 to 25 megacycles per second. These antennas were constructed as part of a design problem of developing an effective radiating system for vertical-incidence ionospheric sounding equipment. The data are in the form of curves, and the antennas are compared with each other from the standpoint of uniformity of impedance over the frequency range. The use of multiple-wire construction to lower the average input impedance, to minimize impedance variations, and to increase radiation efficiency is discussed.
\end{abstract}

\section{General}

The delta antenna (illustrated in figs. 2 and 4) has a number of characteristics that make it attractive as an antenna for vertical-incidence ionospheric work. It is fairly compact, requires only a single large mast, and is easy to erect. However, its input impedance, even when properly terminated, may vary from as low as $300 \mathrm{ohms}$ to as much as 1,200 or 1,400 ohms over the frequency range of 1 to $25 \mathrm{Mc} / \mathrm{s}$, and the use of a transmission line to feed the antenna causes an even greater impedance variation. Furthermore, the radiation efficiency at the lower frequencies of the operating range is quite small.

The vertical rhombic antenna, which is another form of nonresonant antenna frequently used for ionospheric measurements, is somewhat more difficult to construct than the delta, requiring one large mast and two smaller ones. Theoretical considerations lead to the conclusion that the impedance characteristics of a vertical rhombic antenna should be somewhat more uniform than that of a delta. However, the tests performed thus far were limited to existing rhombic antennas that were not designed for optimum impedance characteristics. Theoretical considerations would also lead one to believe that the radiating efficiency of a vertical rhombic antenna, which is small com- pared to the wavelength used, would be lower than a delta or vee antenna designed for the same frequency range.

Elementary considerations indicate that the radiated field of a nonresonant antenna would be proportional to the product of the current flowing through the antenna and the length of the antenna, and is some function of the configuration of the antenna. Because physical limitations restrict the height and, therefore, the length of the antenna, the most promising approach to a more efficient radiator is a multiple wire antenna. Effectively, this lowers the impedance of the antenna, permitting increased current for a given input power. A qualitative analysis indicates that increased radiation efficiency may be thus obtained. Furthermore, because a multiple-wire antenna permits adjustment of wire spacing along its length, it appears to offer a possibility of also securing a more uniform impedance characteristic over the entire frequency range.

The remainder of this report describes the techniques and results of experimental impedance measurements of the following types of antennas:

1. Single-wire delta antennas.

2. A series of multiple-wire delta antennas.

3. A vertical $W$ antenna.

4. A vertical double- $W$ antenna. 


\section{Instrumentation}

For measurement of antenna impedance, a balanced recording impedance meter developed at this Bureau was used. A description of this instrument was presented at the winter 1946-47 IRE convention in New York City. ${ }^{1}$ Figure 1 presents the block diagrams of the instrument.

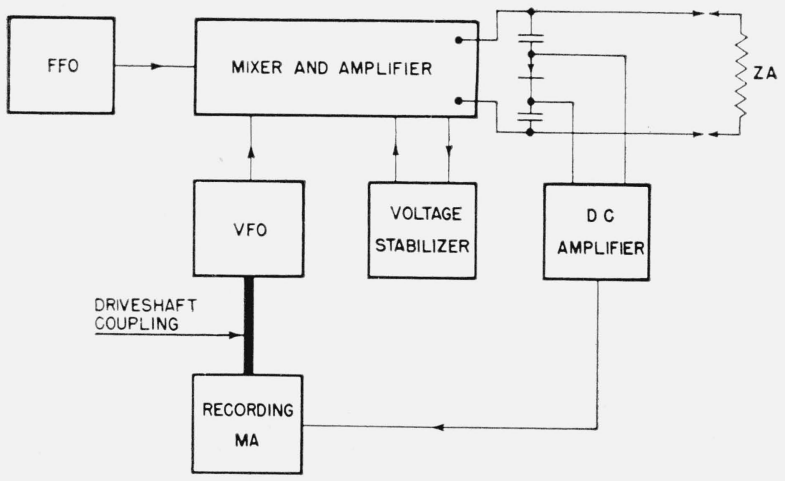

Figure 1. Block diagram of impedance meier.

Briefly, it consists of a $57-\mathrm{Mc} / \mathrm{s}$ fixed-frequency oscillator heating with a variable frequency oscillator covering the frequency band from 57 to $82 \mathrm{Mc} / \mathrm{s}$. The difference frequency of 0 to 25 $\mathrm{Mc} / \mathrm{s}$ is passed through a wide-band amplifier designed to have a constant current output. The output voltage is therefore directly proportional to the absolute magnitude of impedance connected across the output terminals; no direct indication of the phase angle of the impedance has thus far been provided. This voltage is rectified, amplified, and applied to a recording milliammeter. The recording milliammeter and the variable frequency oscillator are mechanically coupled, so that the impedance is plotted as a function of frequency. A voltage stabilizer is incorporated to insure constant output current. The instrument is calibrated by substituting noninductive resistors for the unknown impedance.

\section{Results of Measurements}

Figure 2 shows the construction of a simple form of single-wire delta antenna. The principle of a delta antenna is similar to that of a terminated vertical vee in the higher portion of its frequency range; at lower frequencies it behaves somewhat

\footnotetext{
${ }^{1} \mathrm{H}$. V. Cottony, A method of rapid continuous measurement of antenna impedance over a wide frequency range. Paper presented before IRE Convention, March 6, 1947, New York, N. Y.
}

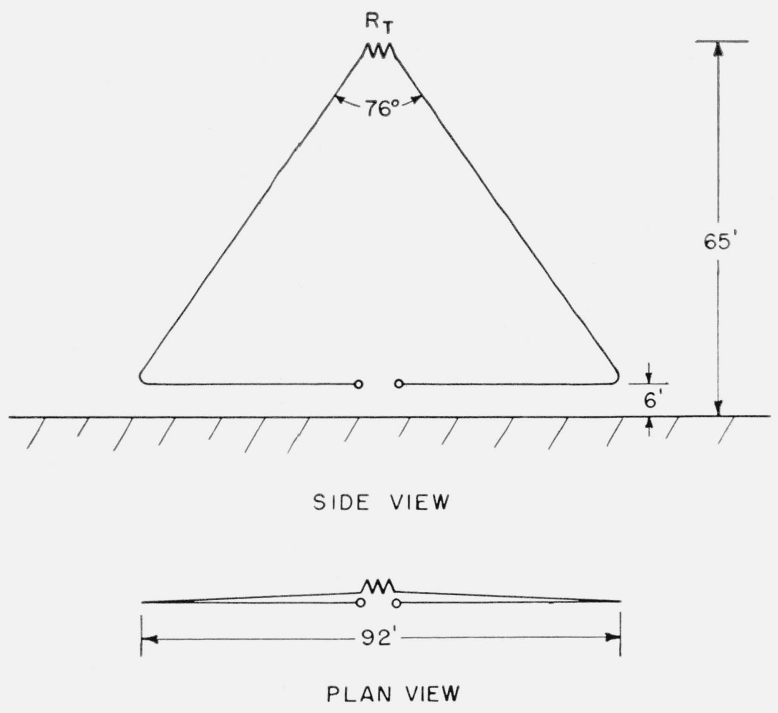

Figure 2. Construction of single-wire delta antenna.

like a loop. The delta construction minimizes the number of supports required and serves to solve the problem of termination. Measurements of the impedance of the antenna shown in figure 2 were made for values of terminating resistance, $R_{T}$, equal to $800,1,000,1,200,1,400$, and $1,600 \mathrm{ohms}$. Figure 3 shows the impedance of the antenna over the frequency range of 1 through $25 \mathrm{Mc} / \mathrm{s}$, using a terminating resistance of $1,000 \mathrm{ohms}$, which was found to be optimum for minimum variation of input impedance. The range of input impedance in this case was from 300 to 1,200 ohms. The value of $R_{T}$ was not particularly critical, since the range of input impedance for the 800-ohm termination was 300 to $1,300 \mathrm{ohms}$, and for the 1,600 -ohm termination was 300 to 1,200 ohms. The impedance at frequencies from 16 to $25 \mathrm{Mc} / \mathrm{s}$ was only slightly affected by changes in terminating resistance. At frequencies below $8 \mathrm{Mc} / \mathrm{s}$, the impedance characteristics were influenced to a

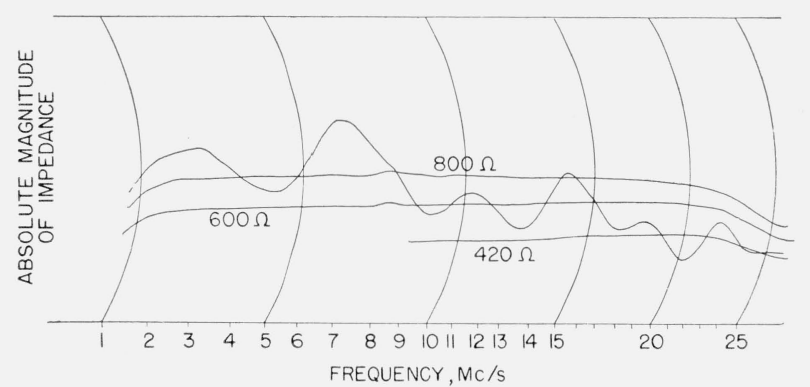

Figure 3. Impedance of single-wire delta antenna for optimum termination of 1,000 ohms. 
much greater degree by different values of terminating resistance, the number and location of the peaks being changed, although the maximum and minimum values of impedance remained about the same.

Figure 4 shows the construction of a variation of the delta antenna designed by J. W. Cox of the Great Baddow Research Laboratories of the British Marconi Co. It is distinguished from the simpler delta by the presence of corkscrew turns at the lower corners of the antenna. These turns are intended to improve the performance of the antenna. Figure 5 shows the impedance characteristics of this antenna. It can be seen that with optimum terminating resistor $\left(R_{T}\right.$ equal to 1,200 ohms), the impedance varied from less than 400 ohms to approximately $1,100 \mathrm{ohms}$, and it appears that the impedance characteristics of the antenna were not appreciably improved by the addition of the corkscrew turns.
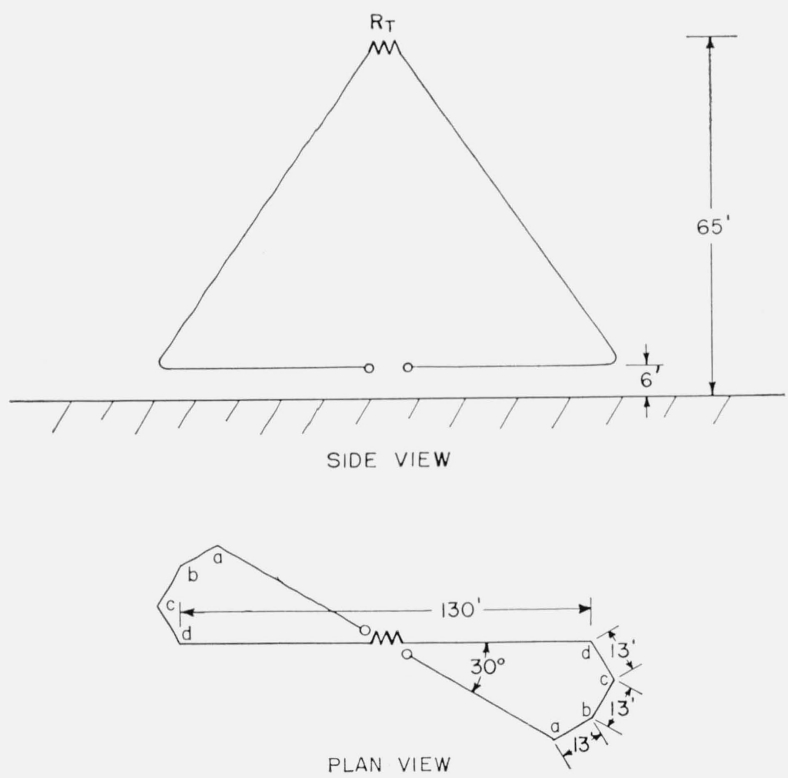

Figure 4. Construction of modified delta antenna.

Point a, $6 \mathrm{ft}$. above ground; point b, $8 \mathrm{ft}$. above ground; point c, $10 \mathrm{ft}$. above ground; point d, $14 \mathrm{ft}$. above ground.

The first multiple-wire delta constructed was identical in size shape to the antenna shown in figure 2, except that it was constructed by using two parallel wires spaced 15 in. apart in place of the single wire. Measurements were made by using a number of different terminations. Figure 6 shows the impedance characteristics of this antenna for an optimum termination of $600 \mathrm{ohms}$.

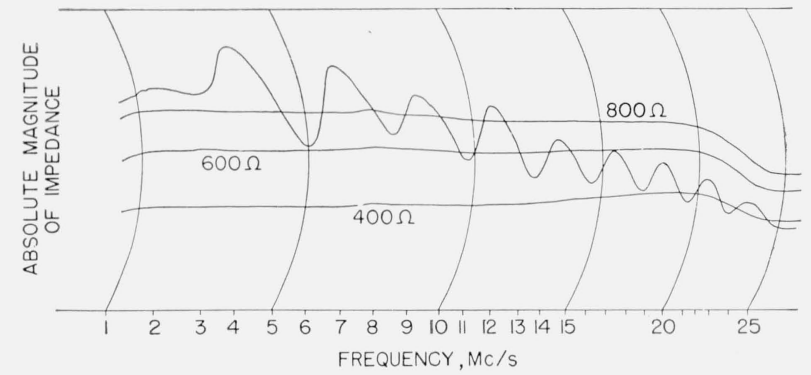

Figure 5. Impedance of modified delta antenna for optimum termination of 1,200 ohms.

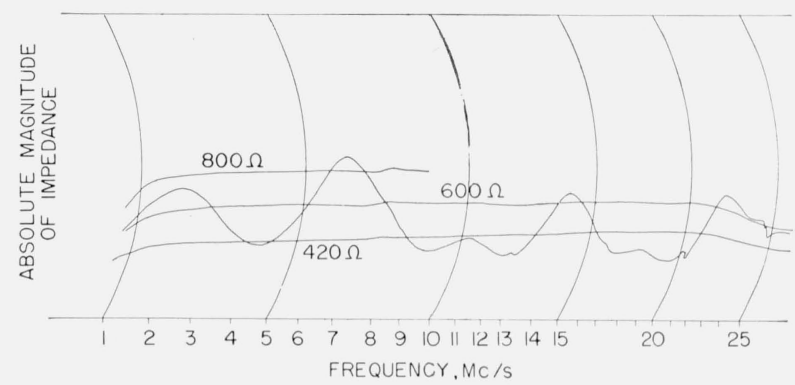

Figure 6. Impedance of parallel-wire delta antenna.

Construction identical to that of figure 2, except that two parallel wires spaced 15 in. apart are used. $\mathrm{R}_{T}=600 \mathrm{ohms}$.

The impedance in this case varied from 300 to 850 ohms. It should be noted that the parallelwire delta terminated with $600 \mathrm{ohms}$ was superior from the standpoint of uniformity of impedance to the single-wire delta terminated with 1,000 ohms. The impedance is lower and the variation small enough so that it would be practical to use a $600-\mathrm{ohm}$ line to feed it.

Several other types of multiple-wire deltas were constructed. The best results were obtained when the oblique wires had the configuration shown in figure 7. Each leg consisted of two wires joined at the apex and the lower corners and flayed 36 in. apart at a point $18 \mathrm{ft}$. from the lower corner. The exact position of the spreaders is not critical nor is the plane of the wires. The horizontal wires, however, have a very significant effect on the impedance characteristics of the antenna. Tests were, therefore, made in which the arrangement of oblique wires shown in figure 7 remained unchanged and with the various arrangements of horizontal wires shown in the same figure. Referring to figure 8, curve A shows the impedance when only a single No. 12 wire was used on each side. Curve B shows the results obtained by replacing the single wire by two No. 18 wires spaced 

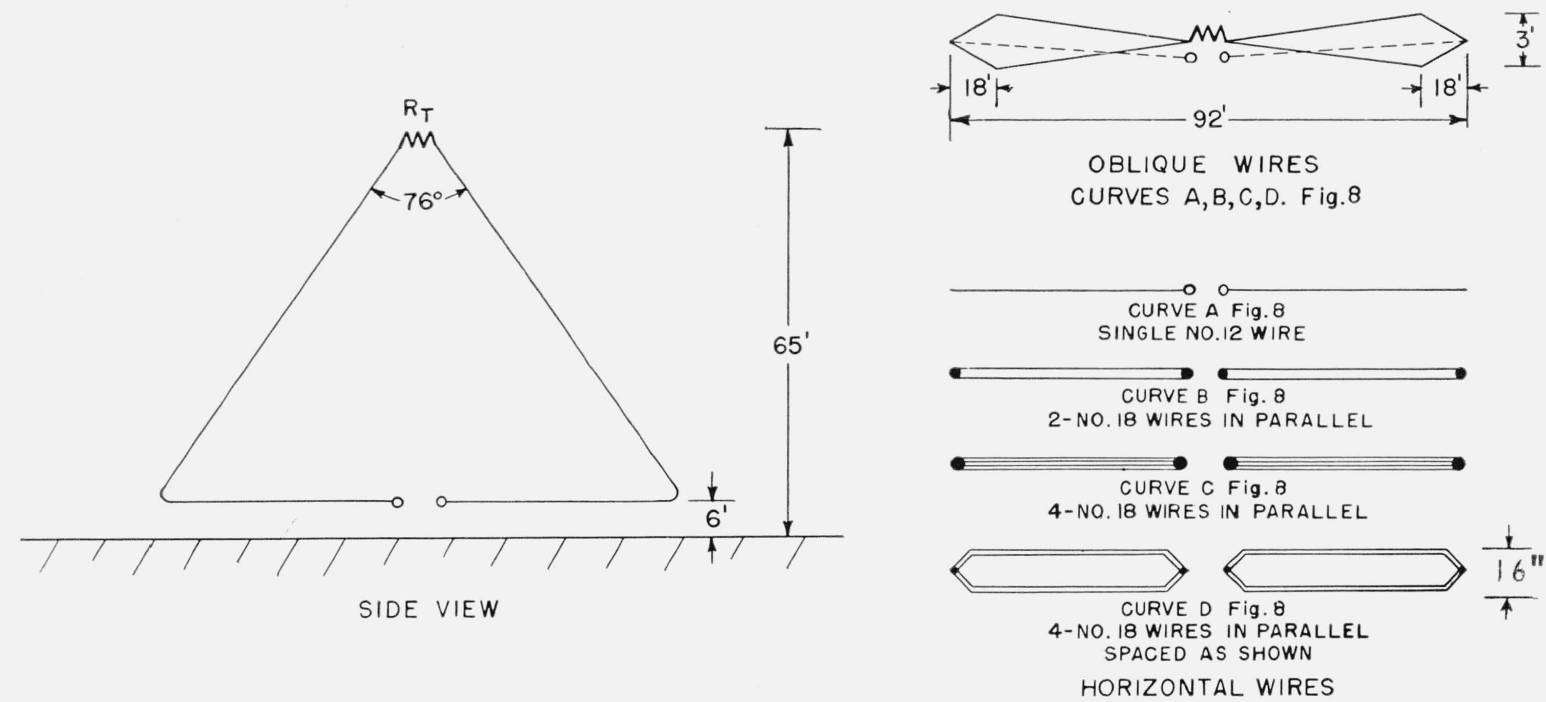

Figure 7. Configuration of oblique and horizontal wires of multiple-wire delta antennas. Impedance curves shown in figure 8.

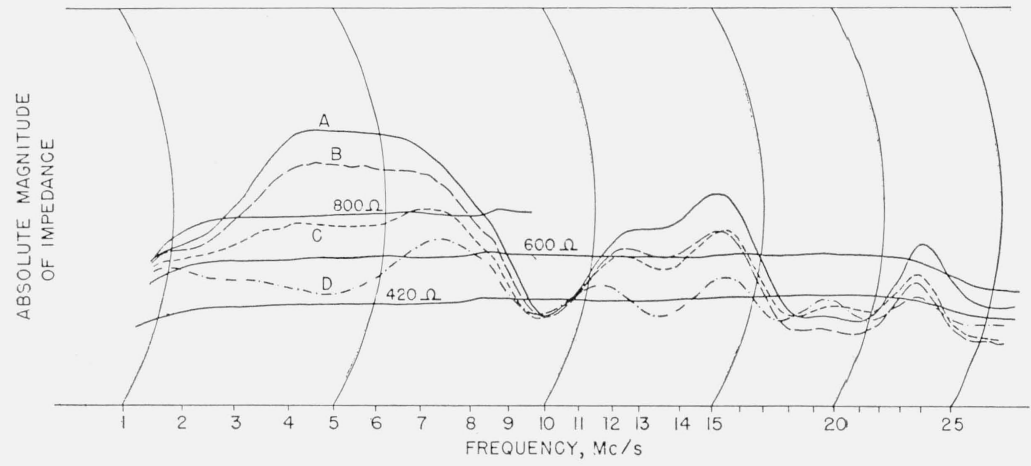

Figure 8. Impedance of multiple-wire delta antennas.

Each antenna terminated with $800-0 h m$ resistor. Constructional details are shown in figure 7 .

0.3 in. apart and connected in parallel. Curve C shows the results when four No. 18 wires were connected in parallel, and curve $\mathrm{D}$ the results when the bottom wires consisted of two pairs of No. 18 wires connected in parallel and spaced 16 in. between pairs, as shown in figure 7 .

Figure 9 shows that the high-frequency impedance characteristics can be improved at the expense of the low-frequency characteristic by changing the terminating resistance from 800 to 600 ohms.

The $W$ antenna, the construction of which is shown in figure 10 , is essentially two vertical-vee antennas excited in phase. One side of each vee terminates in a common resistor, $R_{T}$, and the other sides are grounded by means of vertical wires connected to ground by another resistor equal to one-half the value of $R_{T}$. The vees are fed by 800 -ohm lines, which join to form a 400 ohm line. The impedance characteristic of the transmission-line system with the 800-ohm lines

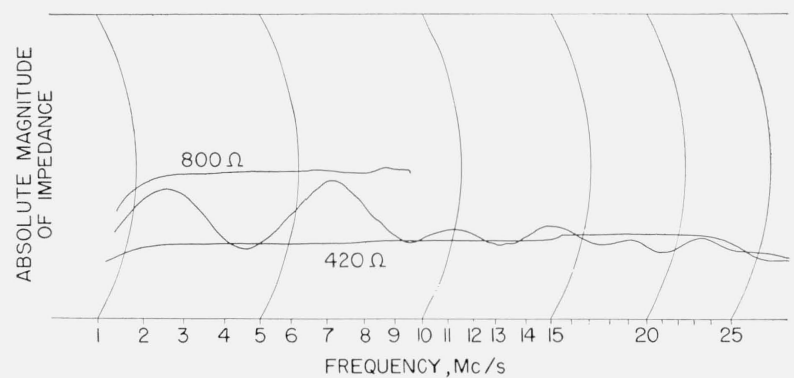

Figure 9. Impedance of multiple-wire delta antenna.

Same as curve D, figure 8 , except that value of terminating resistor is 600 ohms. 

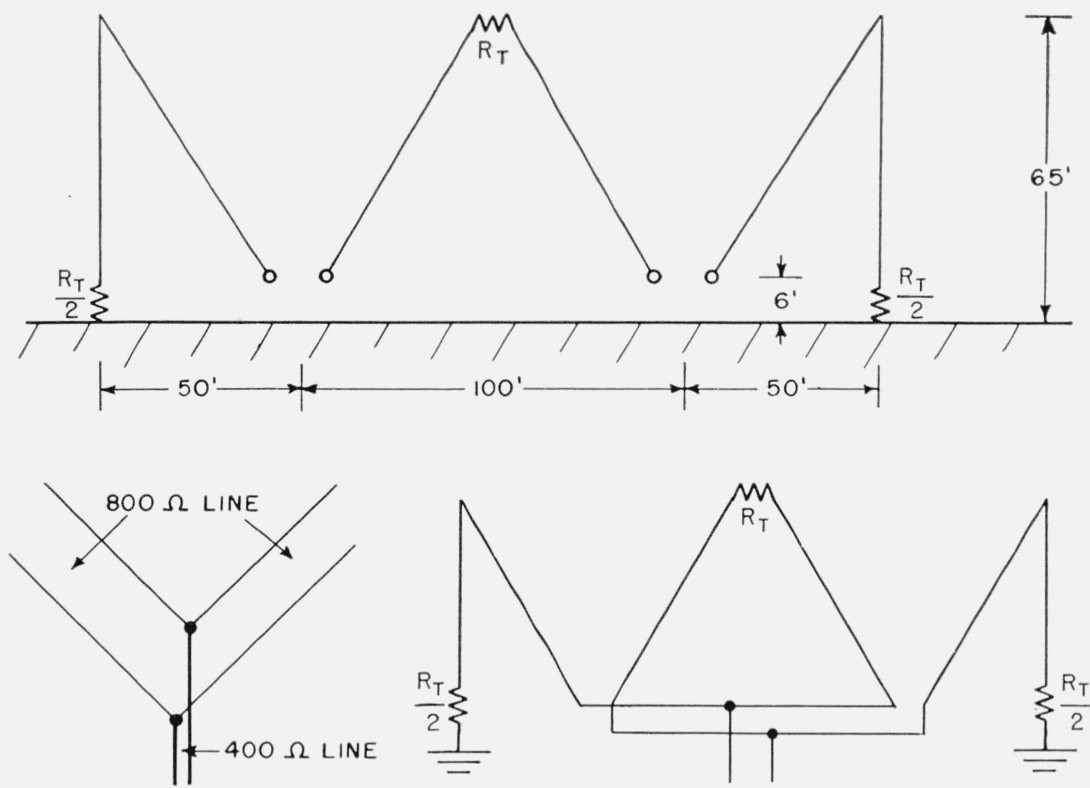

Figure 10. Construction of $W$ antenna and associated transmission line system.

being terminated by 800 -ohm resistors is shown in figure 11. Impedance measurements of the antenna were made for values of $R_{T}$ equal to 600 , $800,1,000,1,200$, and 1,400 ohms. Figure 12 shows the impedance characteristics of this antenna with the optimum termination of 1,000 ohms between the two vee antennas and with 500 ohms on each side. For $R_{T}$ equal to $600 \mathrm{ohms}$, the impedance variation was between 200 and 800 ohms over the entire range. Over most of the range, however, the impedance variation was only about $2: 1$. For $R_{T}$ equal to 1,400 ohms, the impedance variation is only slightly greater than for the 1,000-ohm termination.
Another section of the $W$ antenna was constructed to make a double- $W$ antenna (four vertical vees all excited in phase). This antenna system is shown in figure 13. Each vee was fed with an 800-ohm line, pairs of 800-ohm lines being paralleled and fed with 400-ohm lines. The two resulting 400-ohm lines also were fed in parallel. The impedance looking into the transmission-line system was, therefore, $200 \mathrm{ohms}$. Since $200 \mathrm{ohms}$ is not a suitable load for current designs of multifrequency ionosphere recorders, it was necessary to introduce an impedance transformer. The design of an rf transformer aperiodic over a frequency range of 1 to $25 \mathrm{Mc}$ and capable of handling

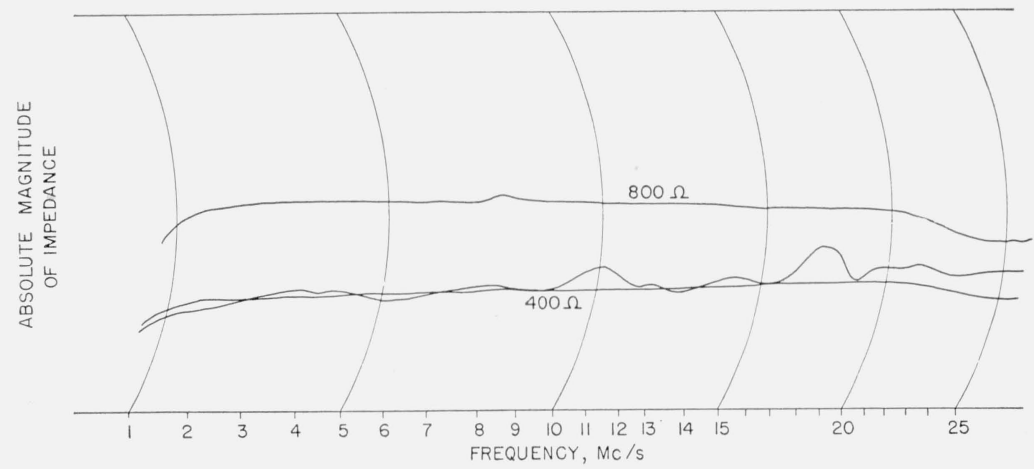

FigURE 11. Impedance of transmission line system used with $W$ antenna. 800-ohm lines terminated with 800-ohm resistors. 


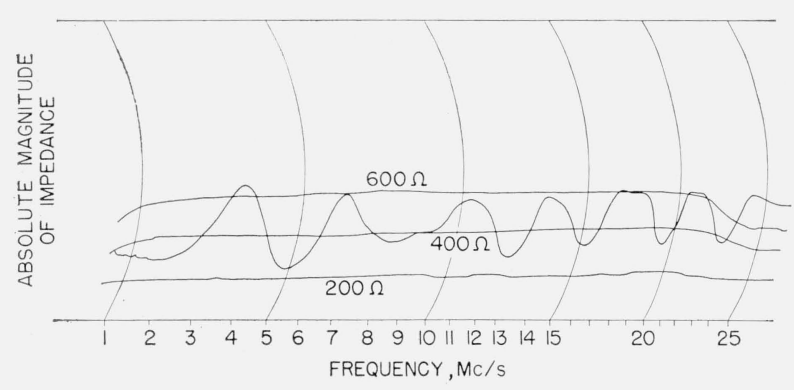

FIGURE 12. Impedance of $W$ antenna for optimum termination of 1,000 ohms. with a pure resistance of 200 ohms is shown in figure 14. Figure 15 shows the impedance characteristic of the entire transmission-line system used with the double- $W$ antenna, including the exponential-line transformer, the 800-ohm lines being terminated with resistance of 800 ohms. Impedance measurements were made of the antenna for values of $R_{T}$ equal to $800,1,000$, and 1,200 ohms. Figure 16 shows the best results obtained with this antenna, which were secured using a 1,000-ohm termination. Over most of
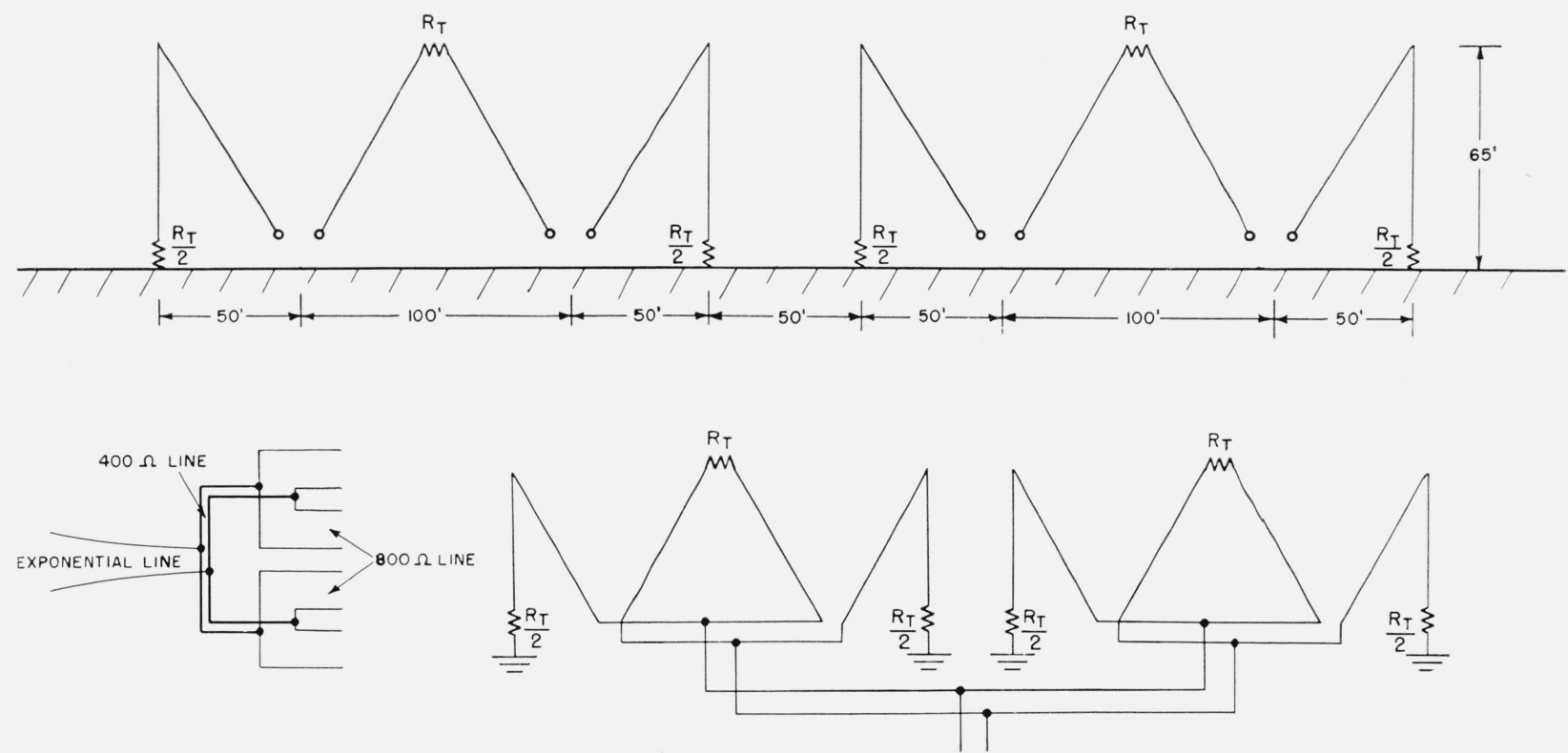

FIGURE 13. Construction of double-W antenna and associated transmission line system.

10 -kw power is quite complicated. The most promising solution appeared to be an exponentialline transformer. ${ }^{2}$ Such a line is reasonably flat at all frequencies substantially greater than the cut-off frequency. Since, for this application, a frequency range of 1 to $25 \mathrm{Mc} / \mathrm{s}$ was desired, the cut-off frequency of the line should be considerably below $1 \mathrm{Mc} / \mathrm{s}$ if impedance variations at the lower frequencies were to be minimized. However, if the cut-off frequency was too low, the line would be inconveniently long.

An exponential line transformer was therefore designed for an impedance transformation of 200 to $600 \mathrm{ohms}$ and for a cut-off frequency of $300 \mathrm{kc}$. The resulting line was $88 \mathrm{~m}$ long. The performance of this line as a transformer when terminated

2 H. A. Wheeler, IR E 27, 65 (Jan. 1939); C. R. Burrows, Bell Sys. Tech. J. 17, 555 (Oct. 1938); W. N. Christiansen, Proc. IRE 34, 576 (June 1947). the range the impedance varied between about 400 and $825 \mathrm{ohms}$. The impedance variation for a value of $R_{T}$ equal to $1,200 \mathrm{ohms}$ was 300 to 1,100 ohms and for a value of $R_{T}$ equal to $800 \mathrm{ohms}$ was from 400 to 900 ohms.

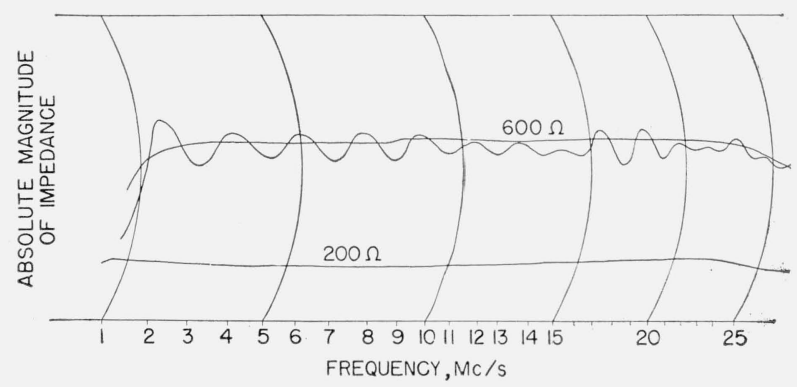

Figure 14. Impedance of exponential transmission line. Line terminated with 200 -ohm resistor. 


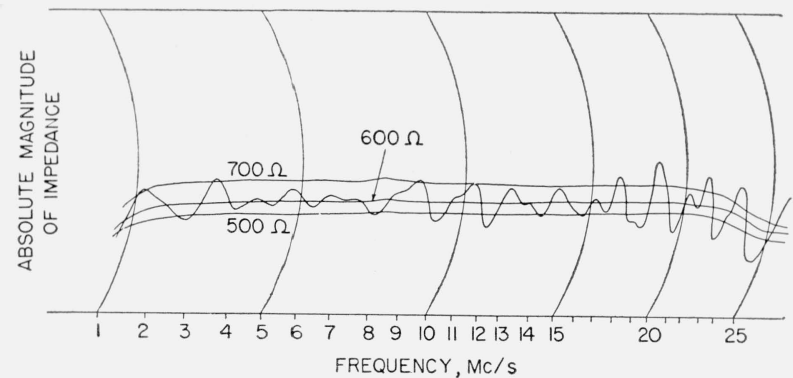

FIGURE 15. Impedance of entire transmission line system used with double $W$ antenna.

800-ohm lines terminated with 800 -ohm resistors.

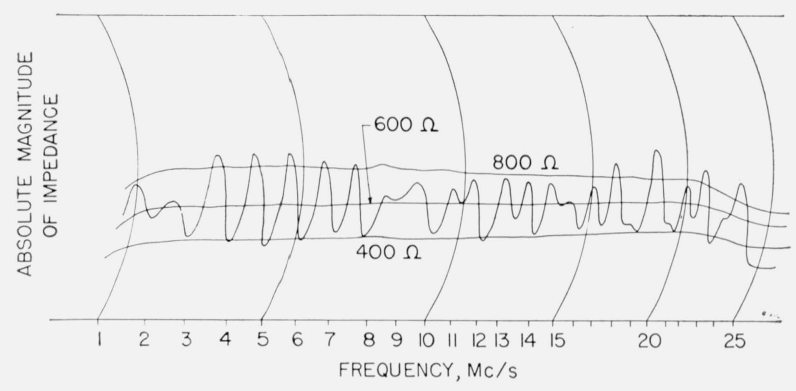

Figure 16. Impedance of double-W antenna for optimum termination of 1,000 ohms.

An attempt was made to determine the approximate radiation efficiency of the antenna system by measuring the power absorbed by the antenna and its associated transmission-line system and the power dissipated in the terminal resistors. This measurement is possible at frequencies at which the impedance of the antenna is a pure resistance. These are the frequencies at which the impedance of the antenna is a maximum of a minimum. The power input to the antenna system is given by the expression

$$
\frac{E_{a}{ }^{2}}{R_{a}} .
$$

The power input to the terminating resistors is given by the sum of

$$
\frac{E_{1}^{2}}{R_{1}}+\frac{E_{2}^{2}}{R_{2}}+\frac{E_{3}^{2}}{R_{3}}+\cdots \frac{E_{r}^{2}}{R_{r}}+\cdots \frac{E_{n}{ }^{2}}{R_{n}} .
$$

In these expressions $R_{a}$ is the value of the input impedance (resistive) of the antenna; $R_{1}, R_{2}$, . . $R_{r} \ldots R_{n}$ are the values of the various terminating resistors, $E_{a}$ is the rms input voltage to the antenna; and $E_{1}, E_{2}, \ldots E_{r} \ldots E_{n}$ are the rms voltages across the respective terminating resistors.

Then the efficiency of the antenna as a radiator is given by the relationship:

$$
\begin{aligned}
& E F F=\frac{\frac{E_{a}{ }^{2}}{R_{a}}-\sum_{r=1}^{r=n} \frac{E_{r}{ }^{2}}{R_{r}}}{E_{a}{ }^{2}} \\
& R_{a} \\
&=1-\frac{R_{a}}{E_{a}{ }^{2}} \sum_{r=1}^{r=n} \frac{E_{r}{ }^{2}}{R_{r}} .
\end{aligned}
$$

It should be noted that the copper, dielectric, and ground losses are all included in the power radiated, and hence the efficiency of the antenna thus obtained is somewhat higher than the true radiation efficiency.

At $3.6 \mathrm{Mc} / \mathrm{s}$, the radiation efficiency of the antenna as measured by this method was found to be approximately 39 percent.

Measurements were made that indicated that each vee had an average input impedance somewhat higher than 800 ohms. An attempt was made to reduce this impedance by constructing the vees with spaced parallel wires, 6 -in. spacing being used. The results are shown in figure 17 . Comparing figures 16 and 17 , it can be seen that, considering the entire frequency range, the singlewire double- $W$ antenna is more desirable. However, for certain limited frequency ranges, the parallel-wave antenna has smaller impedance variations.

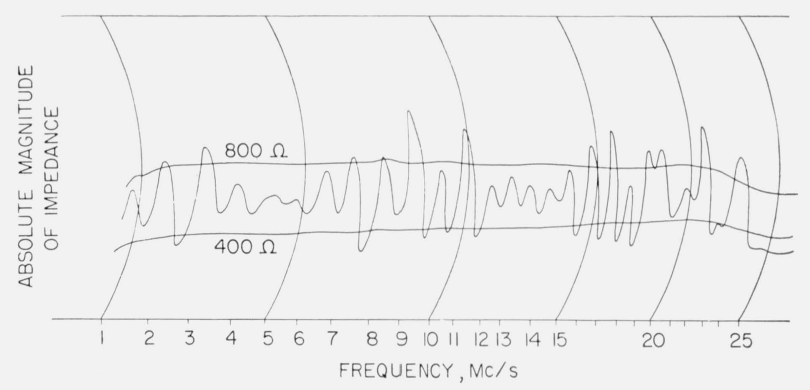

Figure 17. Impedance of parallel wire double-W antenna.

\section{Conclusions}

1. The input impedance of the delta antenna over the frequency range 1 to $25 \mathrm{Mc} / \mathrm{s}$ can be lowered and variations minimized, to the point where it is practical to use a transmission line to feed it, by the use of multiple wires in its construction. 
2. The size and spacing of the wires parallel to the ground have a decided effect on the input impedance of the multiple-wire delta antenna.

3 . The input impedance variations of the experimental double- $W$ antenna were of the same order of magnitude as those obtained with the single-wire delta. However, preliminary measurements indicate that the radiation efficiency of the double- $W$ antenna is somewhat higher than that of the single-wire delta antenna, particularly at the lower frequencies. The double- $W$ antenna is, however, physically large, and its radiation pattern has not yet been determined.

Washington, March 31, 1949. 